University of Nebraska - Lincoln

DigitalCommons@University of Nebraska - Lincoln

\title{
Frequency Shifts in Plate Crystal Resonators Induced by Electric, Magnetic, or Mechanical Fields in Surface Films
}

\author{
Nan Liu \\ University of Nebraska-Lincoln, nliu1@unl.edu \\ J.S. Yang \\ University of Nebraska-Lincoln, jyang1@unl.edu \\ Yuantai Hu \\ Huazhong University of Science and Technology \\ Xuedong Chen \\ Huazhong University of Science and Technology \\ Wei Jiang \\ Huazhong University of Science and Technology, jiangw@hust.edu.cn
}

Follow this and additional works at: https://digitalcommons.unl.edu/mechengfacpub

Part of the Mechanical Engineering Commons

Liu, Nan; Yang, J.S.; Hu, Yuantai; Chen, Xuedong; and Jiang, Wei, "Frequency Shifts in Plate Crystal Resonators Induced by Electric, Magnetic, or Mechanical Fields in Surface Films" (2011). Mechanical \& Materials Engineering Faculty Publications. 75.

https://digitalcommons.unl.edu/mechengfacpub/75

This Article is brought to you for free and open access by the Mechanical \& Materials Engineering, Department of at DigitalCommons@University of Nebraska - Lincoln. It has been accepted for inclusion in Mechanical \& Materials Engineering Faculty Publications by an authorized administrator of DigitalCommons@University of Nebraska Lincoln. 


\title{
Frequency Shifts in Plate Crystal Resonators Induced by Electric, Magnetic, or Mechanical Fields in Surface Films
}

\author{
Nan Liu, Jiashi Yang, Yuantai Hu, Xuedong Chen, and Wei Jiang
}

\begin{abstract}
We study frequency shifts in plate crystal resonators with surface films. The films are multiphysical, including the effects of inertia, stiffness, intrinsic stress, piezoelectric coupling, and piezomagnetic coupling. Mindlin's two-dimensional equations for a crystal plate with two elastic surface films are generalized to include the multiphysical effects of the films. They are used to study thickness-shear vibrations of a rotated Y-cut quartz plate with initial fields resulting from the mechanical, electric, and magnetic fields in the surface films. Frequency shifts caused by the initial fields are calculated and examined. Results show that plate crystal resonators with multiphysical surface films may be used for electric/magnetic field sensing.
\end{abstract}

\section{INTRODUCTION}

$\mathrm{C}$ RYSTAL resonators are key elements of electrical cir$\checkmark$ cuits called oscillators providing frequency standards for time-keeping and frequency operations. Oscillators are important components of telecommunication devices, satellites, radars, and other electronic equipment. These devices are often used in harsh environment or on objects in motion. For these applications, high frequency stability of the crystal resonators against environmental effects like a temperature change or acceleration is desired [1]. During the last few decades, the applications of crystal resonators for sensing grew rapidly [2]-[5]. For sensor applications, the resonant frequencies of crystal resonators must be made sensitive to environmental changes for high sensitivity. Thickness-shear (TSh) vibration modes of a rotated Y-cut quartz plate are the most widely used structure and modes of crystal resonators and sensors.

Either as a resonator or a sensor, a crystal plate is often covered with surface films. These films may be metal electrodes or chemically/biologically selective materials to attract certain substances for sensing. They have their own physical properties. For thin films, the frequency effect of the inertia alone is the foundation of quartz crystal micro-

Manuscript received July 6, 2011; accepted September 1, 2011. This work was supported by the National Science Foundation of China (grant numbers 10872074 and 10932004) and the Major State Basic Research Development Program of China (973 Program; grant number 2009CB724205).

N. Liu and J. Yang are with the Department of Mechanical and Materials Engineering, University of Nebraska, Lincoln, NE.

$\mathrm{Y} . \mathrm{Hu}$ is with the Department of Mechanics, Huazhong University of Science and Technology, Wuhan, China.

$\mathrm{X}$. Chen and W. Jiang are with the National NC System Engineering Research Center, Huazhong University of Science and Technology, Wuhan, China (e-mail: jiangw@hust.edu.cn).

Digital Object Identifier 10.1109/TUFFC.2011.2122 balances, mass sensors used in chemical/biological sensing. When the films are not thin, their stiffness also needs to be considered. The inertia and stiffness of the films are relatively simple effects that can be modeled by the linear theory of elasticity. They have been studied relatively thoroughly [4], [5], and can be easily determined through calibration once a device is made.

There are other frequency effects of the surface films that are more complicated. These include the film intrinsic stresses [6], [7] resulting from manufacturing processes and the deformation of the films caused by electric or magnetic fields when the films have piezoelectric/piezomagnetic couplings [8], [9]. The intrinsic stresses and multiphysical couplings in the films manifest themselves in a crystal resonator through the stresses and strains they produce, called initial or biasing fields in resonators. When biasing fields are present, the resonator frequencies are slightly different from those without biasing fields. The behavior of crystal resonators with the presence of biasing fields is governed by the theory for small fields superposed on a bias [10] which needs to be derived from a nonlinear theory [11]. Because of the complexity of these theories, the effects of biasing fields in resonators resulting from surface films are rarely studied. Film intrinsic stress and thermal expansion were treated separately in [7] and [12] for resonator applications.

Recently it has been shown through simple, one-dimensional analyses that plate crystal resonators structurally integrated with piezoelectric/piezomagnetic films possessing strong piezoelectric/piezomagnetic couplings may be considered for electric/magnetic field sensing [13], and that it is possible to use surface piezoelectric films to manipulate frequency shifts in crystal resonators caused by other effects, e.g., accelerations [14]. A crystal plate with a layer of a different material is also useful in other frequency designs of resonators, e.g., in temperature [15] or other compensation techniques in which frequency shifts from different origins are used to cancel each other out.

In this paper, we explore further the ideas of electric/ magnetic field sensing and frequency manipulation in [13] and [14]. To predict the behavior of real devices of finite sizes, we analyze the more realistic situation of finite, two-dimensional plates for frequency shifts caused by multiphysical effects in surface films. For this purpose, we generalize an earlier version of first-order, two-dimensional equations by Mindlin [16] for coupled extension, TSh, and flexural vibrations of a crystal plate with elastic surface films (see Fig. 1) by including intrinsic stresses and piezo- 


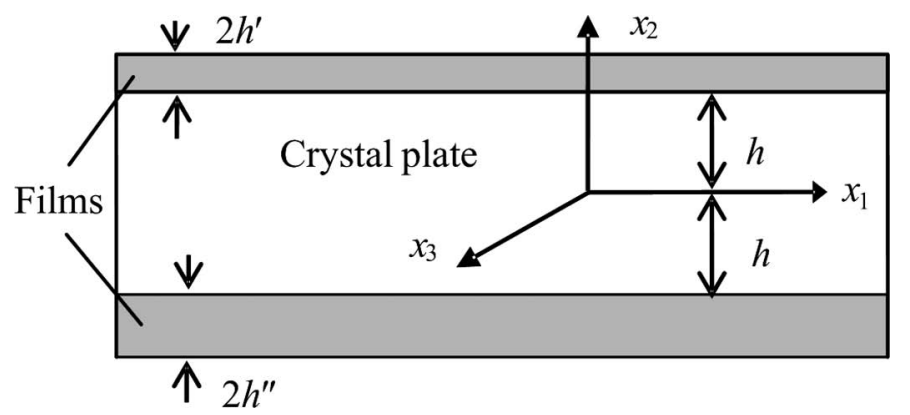

Fig. 1. A crystal plate with asymmetric surface films.

electric/piezomagnetic couplings in the films. The equations are then reduced to the case of coupled extension and elementary flexure without shear deformations, and are used to determine the biasing fields induced by the surface films. With these biasing fields found, frequency shifts caused by electric, magnetic, and intrinsic stresses in the films are calculated and examined.

\section{Two-Dimensional Equations for a CRYstal Plate With Surface Films}

In this section, we generalize the two-dimensional equations for crystal plates with elastic films in [16] to include multiphysical effects in the films. Although the generalization is only in the constitutive relations of the films and is straightforward, it is somewhat tedious, and a concise but complete presentation is given because [16] is not conveniently available.

\section{A. Equations for the Crystal Plate}

We begin with a summary of the two-dimensional equations for coupled extensional, flexural, and TSh vibrations of a quartz crystal plate alone without surface films. The very small piezoelectric coupling in quartz is neglected, as is usual in frequency analysis of quartz devices. Cartesian tensor notation is employed, with the summation convention for repeated indices. A comma followed by an index denotes a partial derivative with respect to the coordinate associated with the index. $i, j, k$, and $l$ take the values 1 , 2 , and $3 ; a, b, c$, and $d$ take the values 1 and 3 but not 2 . Consider the crystal plate of thickness $2 h$ in Fig. $1 . x_{1}$ and $x_{3}$ are in the middle plane. $x_{2}$ is along the plate normal. The components of displacement, $u_{i}$, are approximated by the first two terms of a power series in $x_{2}$ :

$$
u_{i}=u_{i}^{(0)}\left(x_{1}, x_{3}, t\right)+x_{2} u_{i}^{(1)}\left(x_{1}, x_{3}, t\right)
$$

and the components of stress, $T_{i j}$, by the stress-resultants, $T_{i j}^{(0)}$, and stress couples, $T_{i j}^{(1)}$ :

$$
T_{i j}^{(0)} \equiv \int_{-h}^{h} T_{i j} \mathrm{~d} x_{2}, \quad T_{i j}^{(1)} \equiv \int_{-h}^{h} x_{2} T_{i j} \mathrm{~d} x_{2} .
$$

The stress-equations of motion, $T_{i j, i}=\rho \ddot{u}_{j}$, are approximated by the following equations:

$$
\begin{gathered}
T_{i j, i}^{(0)}+F_{j}^{(0)}=2 h \rho \ddot{u}_{j}^{(0)}, \quad i, j=1,2,3, \\
T_{a b, a}^{(1)}-T_{2 b}^{(0)}+F_{b}^{(1)}=\frac{2}{3} h^{3} \ddot{\rho} u_{b}^{(1)}, \quad a, b=1,3,
\end{gathered}
$$

where a dot denotes time derivative, $F_{j}^{(0)}$ and $F_{b}^{(1)}$ are surface loads defined by

$$
\begin{gathered}
\left.F_{j}^{(0)} \equiv T_{2 j}\right|_{h}-\left.T_{2 j}\right|_{-h}, \\
\left.F_{b}^{(1)} \equiv h T_{2 b}\right|_{h}+\left.h T_{2 b}\right|_{-h},
\end{gathered}
$$

and $\rho$ is the mass density. The stress-strain relations, $T_{i j}$ $=c_{i j k l} S_{k l}$, are approximated by

$$
\begin{aligned}
& T_{i j}^{(0)}=2 h g_{i j k l}^{*} S_{k l}^{(0)}, \\
& T_{a b}^{(1)}=\frac{2}{3} h^{3} \gamma_{a b c d} S_{c d}^{(1)},
\end{aligned}
$$

where

$$
\begin{aligned}
g_{i j k l}^{*} & \equiv \kappa_{i+j-2}^{m} \kappa_{k+l-2}^{n} g_{i j k l}, \quad \text { (not summed) } \\
g_{i j k l} & \equiv c_{i j k l}-c_{i j 22} c_{22 k l} / c_{2222} \\
\gamma_{a b c d} & \equiv c_{a b c d}-c_{a b 2 j} c_{2 k c d}\left(c_{2 j 2 k}\right)^{-1}
\end{aligned}
$$

$g_{i j k l}$ are obtained from the stress relaxation $T_{22}^{(0)}=0$. They are then modified into $g_{i j k l}^{*}$ by the shear correction factors $\kappa_{1}$ and $\kappa_{3}$ [16], [17]. $\gamma_{a b c d}$ is obtained from the stress relaxation $T_{2 j}^{(1)}=0$. In (7), $m$ and $n$ are given by

$$
m=\cos ^{2}(i j \pi / 2), \quad n=\cos ^{2}(k l \pi / 2) .
$$

The values of $\kappa_{a}$ will be provided later. The strains in (6) are given in terms of the displacements by

$$
\begin{gathered}
S_{i j}^{(0)}=\frac{1}{2}\left(u_{i, j}^{(0)}+u_{j, i}^{(0)}+\delta_{2 j} u_{i}^{(1)}+\delta_{2 i} u_{j}^{(1)}\right), \\
S_{a b}^{(1)}=\frac{1}{2}\left(u_{a, b}^{(1)}+u_{b, a}^{(1)}\right),
\end{gathered}
$$

in which $\delta_{i j}$ is the Kronecker symbol.

\section{B. Equations for the Films}

Fields associated with the upper and lower films will be designated by primes and double primes, respectively. The film thickness, $2 h^{\prime}$ and $2 h^{\prime \prime}$, are usually much thinner than the crystal plate. Therefore, the films are modeled by the equations of extension. The bending and twisting moments, $T_{a b}^{\prime(1)}$ and $T_{a b}^{\prime \prime(1)}$, and the TSh stress-resultants, $T_{a 2}^{\prime(0)}$ and $T_{a 2}^{\prime \prime(0)}$, may be neglected in the films. Thus, the equations for the upper film are

$$
\begin{gathered}
\delta_{j b}^{0} T_{a b, a}^{\prime(0)}+F_{j}^{\prime(0)}=2 h^{\prime} \rho^{\prime} \ddot{u}_{j}^{\prime(0)}, \\
\left.F_{j}^{\prime(0)} \equiv T_{2 j}^{\prime}\right|_{h^{\prime}}-\left.T_{2 j}^{\prime}\right|_{-h^{\prime}}, \\
T_{a b}^{\prime(0)}=2 h^{\prime}\left(\tau_{a b}^{\prime}+\gamma_{a b c d}^{\prime} S_{c d}^{\prime(0)}-e_{k a b}^{\prime} E_{k}^{\prime}-h_{k a b}^{\prime} H_{k}^{\prime}\right),
\end{gathered}
$$




$$
S_{a b}^{\prime(0)}=\frac{1}{2}\left(u_{a, b}^{\prime(0)}+u_{b, a}^{\prime(0)}\right)
$$

and for the lower film,

$$
\begin{gathered}
\delta_{j b}^{0} T_{a b, a}^{\prime \prime(0)}+F_{j}^{\prime \prime(0)}=2 h^{\prime \prime} \rho^{\prime \prime} \ddot{u}_{j}^{\prime \prime(0)}, \\
\left.F_{j}^{\prime \prime(0)} \equiv T_{2 j}^{\prime \prime}\right|_{h^{\prime \prime}}-\left.T_{2 j}^{\prime \prime}\right|_{-h^{\prime \prime}}, \\
T_{a b}^{\prime \prime(0)}=2 h^{\prime \prime}\left(\tau_{a b}^{\prime \prime}+\gamma_{a b c d^{\prime \prime}}^{\prime \prime} S_{c d}^{\prime \prime(0)}-e_{k a b}^{\prime \prime} E_{k}^{\prime \prime}-h_{k a b}^{\prime \prime} H_{k}^{\prime \prime}\right), \\
S_{a b}^{\prime \prime(0)}=\frac{1}{2}\left(u_{a, b}^{\prime \prime(0)}+u_{b, a}^{\prime \prime(0)}\right),
\end{gathered}
$$

where $\delta_{j b}^{0}=\delta_{c b}$ when $j=c$ and $\delta_{j b}^{0}=0$ when $j=2$. Eqs. (12) and (16) are more general than the corresponding ones in [16] because of the inclusion of the thin-film intrinsic stress $\tau_{a b}^{\prime}$, the electric field $E_{k}^{\prime}$, and the magnetic field $H_{k}^{\prime} . e_{k a b}^{\prime}$ and $h_{k a b}^{\prime}$ are thin-film piezoelectric and piezomagnetic constants. They are obtained from the stress relaxation $T_{2 j}^{(0)}=0$. We assume that the intrinsic stress $\tau_{a b}^{\prime}$, the strain $S_{c d}^{\prime(0)}$, the electric field $E_{k}^{\prime}$, and the magnetic field $H_{k}^{\prime}$ are all infinitesimal. Only their first-order effects on the resonant frequencies are of interest. $\tau_{a b}^{\prime}, E_{k}^{\prime}$, and $H_{k}^{\prime}$ are considered known and they act as loads on the structure. For the lower film, the situation is similar.

\section{Equations for the Crystal Plate With Surface Films}

The equations of the crystal plate and the surface films are joined together using the following interface continuity conditions of tractions and displacements:

$$
\begin{gathered}
\left.T_{2 j}^{\prime}\right|_{-h^{\prime}}=\left.T_{2 j}\right|_{h},\left.\quad T_{2 j}^{\prime \prime}\right|_{h^{\prime \prime}}=\left.T_{2 j}\right|_{-h}, \\
u_{i}^{\prime(0)}=u_{i}^{(0)}+h \delta_{i a}^{0} u_{a}^{(1)}, \quad u_{i}^{\prime(0)}=u_{i}^{(0)}-h \delta_{i a}^{0} u_{a}^{(1)} .
\end{gathered}
$$

Through (18), the surface loads of the crystal plate, $F_{j}^{(0)}$ and $F_{b}^{(1)}$ in (3) and (4), may be expressed in terms of the surface loads $F_{j}^{\prime(0)}$ and $F_{j}^{\prime \prime(0)}$ of the films. Then the stress equations of motion, (10) and (14), of the films may be incorporated into (3) and (4) by substitution, thus forming five stress-equations of motion of the crystal plate with the films. In these equations, the accelerations may be expressed in terms of $u_{i}^{(0)}$ and $u_{a}^{(1)}$ by means of (19). The stress equations of motion may then be converted to five displacement equations of motion for the five displacements, $u_{i}^{(0)}$ and $u_{a}^{(1)}$, through the use of the three sets of stress-strain-displacement relations and (19). This is carried out in the following.

From (5a) and (18), and from (5b) and (18), we obtain

$$
\begin{gathered}
F_{j}^{(0)}=\left.T_{2 j}^{\prime}\right|_{-h^{\prime}}-\left.T_{2 j}^{\prime \prime}\right|_{h^{\prime \prime}}, \\
F_{b}^{(1)}=\left.h T_{2 b}^{\prime}\right|_{-h^{\prime}}+\left.h T_{2 b}^{\prime \prime}\right|_{h^{\prime \prime}} .
\end{gathered}
$$

From the sum and difference of (11) and (15),

$$
\left.T_{2 j}^{\prime}\right|_{-h^{\prime}}-\left.T_{2 j}^{\prime \prime}\right|_{h^{\prime \prime}}=F_{j}^{(0)}-F_{j}^{\prime(0)}-F_{j}^{\prime \prime(0)},
$$

$$
\left.h T_{2 b}^{\prime}\right|_{-h^{\prime}}+\left.h T_{2 b}^{\prime \prime}\right|_{h^{\prime \prime}}=F_{b}^{(1)}-h\left(F_{b}^{\prime(0)}-F_{b}^{\prime \prime(0)}\right),
$$

where

$$
\begin{gathered}
\left.F_{j}^{(0)} \equiv T_{2 j}^{\prime}\right|_{h^{\prime}}-\left.T_{2 j}^{\prime \prime}\right|_{-h^{\prime \prime}}, \\
\left.F_{b}^{(1)} \equiv h T_{2 b}^{\prime}\right|_{h^{\prime}}+\left.h T_{2 b}^{\prime \prime}\right|_{-h^{\prime \prime}},
\end{gathered}
$$

are the loads on the outer surfaces of the films. Hence, from $(20)-(23)$,

$$
\begin{aligned}
& F_{j}^{(0)}=F_{j}^{(0)}-\left(F_{j}^{\prime(0)}+F_{j}^{\prime \prime(0)}\right), \\
& F_{b}^{(1)}=F_{b}^{(1)}-h\left(F_{b}^{\prime(0)}-F_{b}^{\prime \prime(0)}\right) .
\end{aligned}
$$

From the sum and difference of (10) and (14),

$$
\begin{gathered}
F_{j}^{\prime(0)}+F_{j}^{\prime \prime(0)}=-\delta_{j b}^{0}\left(T_{a b}^{\prime(0)}+T_{a b}^{\prime \prime(0)}\right)_{, a}+2 h^{\prime} \rho^{\prime} \ddot{u}_{j}^{\prime(0)} \\
+2 h^{\prime \prime} \rho^{\prime \prime} \ddot{u}_{j}^{\prime \prime(0)}, \\
F_{b}^{\prime(0)}-F_{b}^{\prime \prime(0)}=-\left(T_{a b}^{\prime(0)}-T_{a b}^{\prime \prime(0)}\right)_{, a}+2 h^{\prime} \rho^{\prime} \ddot{u}_{b}^{\prime(0)}-2 h^{\prime \prime} \rho^{\prime \prime} \ddot{u}_{b}^{\prime \prime(0)} .
\end{gathered}
$$

Substituting these expressions into (26) and (27), and using (19), we have

$$
\begin{aligned}
F_{j}^{(0)}= & F_{j}^{(0)}+\delta_{j b}^{0}\left(T_{a b}^{\prime(0)}+T_{a b}^{\prime \prime(0)}\right)_{, a}-2 h \rho R_{\mathrm{S}} \ddot{u}_{j}^{(0)} \\
& -2 h^{2} \rho R_{\mathrm{D}} \delta_{j a}^{0} \ddot{u}_{a}^{(1)}, \\
F_{b}^{(1)}=F_{b}^{(1)}+ & h\left(T_{a b}^{\prime(0)}-T_{a b}^{\prime \prime(0)}\right)_{, a}-2 h^{3} \rho R_{\mathrm{S}} \ddot{u}_{b}^{(1)}-2 h^{2} \rho R_{\mathrm{D}} \ddot{u}_{b}^{(0)},
\end{aligned}
$$

where we have denoted

$$
R_{\mathrm{S}}=\left(\rho^{\prime} h^{\prime}+\rho^{\prime \prime} h^{\prime \prime}\right) / \rho h, \quad R_{\mathrm{D}}=\left(\rho^{\prime} h^{\prime}-\rho^{\prime \prime} h^{\prime \prime}\right) / \rho h .
$$

$R_{\mathrm{S}}$ and $R_{\mathrm{D}}$ are the mass ratios of the sum and difference of the films to the crystal plate.

Finally, substituting (30) and (31) into (3) and (4), we arrive at the following stress equations of motion of the crystal plate carrying the films:

$$
\begin{gathered}
\mathcal{T}_{i j, i}^{(0)}+F_{j}^{(0)}=2 h \rho\left(1+R_{\mathrm{S}}\right) \ddot{u}_{j}^{(0)}+2 h^{2} \rho R_{\mathrm{D}} \delta_{j b}^{0} \ddot{u}_{b}^{(1)}, \\
\mathcal{T}_{a b, a}^{(1)}-\mathcal{T}_{2 b}^{(0)}+F_{b}^{(1)}=\frac{2}{3} h^{3} \rho\left(1+3 R_{\mathrm{S}}\right) \ddot{u}_{b}^{(1)}+2 h^{2} \rho R_{\mathrm{D}} \ddot{u}_{b}^{(0)},
\end{gathered}
$$

where the total resultants including contributions from the plate and the films are

$$
\begin{gathered}
\mathcal{T}_{i j}^{(0)} \equiv T_{i j}^{(0)}+\delta_{i a}^{0} \delta_{j b}^{0}\left(T_{a b}^{\prime(0)}+T_{a b}^{\prime \prime(0)}\right) \\
\mathcal{T}_{a b}^{(1)} \equiv T_{a b}^{(1)}+h\left(T_{a b}^{\prime(0)}-T_{a b}^{\prime \prime(0)}\right)
\end{gathered}
$$

Substituting into (35) and (36) the constitutive relations (6), (12), and (16), and then the strain-displacement relations (13) and (17), we find, after using (19) and then (9), the following constitutive relations for the plate including the films: 
$\tau_{i j}^{(0)}=2 h g_{i j k l}^{*} S_{k l}^{(0)}+2 h \delta_{i a}^{0} \delta_{j b}^{0}\left(\gamma_{a b c d}^{\mathrm{S}} S_{c d}^{(0)}+h \gamma_{a b c d}^{\mathrm{D}} S_{c d}^{(1)}\right)+2 h N_{i j}$

$\tau_{a b}^{(1)}=\frac{2}{3} h^{3} \gamma_{a b c d} S_{c d}^{(1)}+2 h^{3} \gamma_{a b c d}^{\mathrm{S}} S_{c d}^{(1)}+2 h^{2} \gamma_{a b c d}^{\mathrm{D}} S_{c d}^{(0)}+2 h M_{a b}$,

where we have defined

$$
\begin{gathered}
N_{i j}=\delta_{i a}^{0} \delta_{j b}^{0} \frac{h^{\prime}}{h}\left(\tau_{a b}^{\prime}-e_{k a b}^{\prime} E_{k}^{\prime}-h_{k a b}^{\prime} H_{k}^{\prime}\right) \\
+\delta_{i a}^{0} \delta_{j b}^{0} \frac{h^{\prime \prime}}{h}\left(\tau_{a b}^{\prime \prime}-e_{k a b}^{\prime \prime} E_{k}^{\prime \prime}-h_{k a b}^{\prime \prime} H_{k}^{\prime \prime}\right), \\
M_{a b}=h^{\prime}\left(\tau_{a b}^{\prime}-e_{k a b}^{\prime} E_{k}^{\prime}-h_{k a b}^{\prime} H_{k}^{\prime}\right) \\
-h^{\prime \prime}\left(\tau_{a b}^{\prime \prime}-e_{k a b}^{\prime \prime} E_{k}^{\prime \prime}-h_{k a b}^{\prime \prime} H_{k}^{\prime \prime}\right), \\
\gamma_{a b c d}^{\mathrm{S}} \equiv\left(h^{\prime} \gamma_{a b c d}^{\prime}+h^{\prime \prime} \gamma_{a b c d}^{\prime \prime}\right) / h, \\
\gamma_{a b c d}^{\mathrm{D}} \equiv\left(h^{\prime} \gamma_{a b c d}^{\prime}-h^{\prime \prime} \gamma_{a b c d}^{\prime \prime}\right) / h .
\end{gathered}
$$

$N_{i j}$ and $M_{a b}$ describe the contributions from the films to the extension and bending of the plate. The displacement equations of motion may be obtained by substituting (9) into (37) and (38) and then substituting (37) and (38) into (33) and (34), resulting in:

$$
\begin{gathered}
g_{i j k l}^{*}\left(u_{k, l i}^{(0)}+\delta_{2 k} u_{l, i}^{(1)}\right)+\delta_{j b}^{0} \gamma_{a b c d}^{\mathrm{S}} u_{c, d a}^{(0)}+h \delta_{j b}^{0} \gamma_{a b c d}^{\mathrm{D}} u_{c, d a}^{(1)} \\
+\frac{1}{2} h^{-1} \mathcal{F}_{j}^{(0)}+N_{i j, i}=\rho\left(1+R_{\mathrm{S}}\right) \ddot{u}_{j}^{(0)}+h \rho R_{\mathrm{D}} \delta_{j a}^{0} \ddot{u}_{a}^{(1)} \\
\frac{1}{3} h^{2} \gamma_{a b c d} u_{c, d a}^{(1)}-g_{2 b k l}^{*}\left(u_{k, l}^{(0)}+\delta_{2 k} u_{l}^{(1)}\right)+h^{2} \gamma_{a b c d}^{\mathrm{S}} u_{c, d a}^{(1)} \\
+h \gamma_{a b c d}^{\mathrm{D}} u_{c, d a}^{(0)}+\frac{1}{2} h^{-1} \mathcal{F}_{b}^{(1)}+M_{a b, a} \\
=\frac{1}{3} h^{2} \rho\left(1+3 R_{\mathrm{S}}\right) \ddot{u}_{b}^{(1)}+h \rho R_{\mathrm{D}} \ddot{u}_{b}^{(0)} .
\end{gathered}
$$

Finally, the shear correction factors $\kappa_{1}$ and $\kappa_{3}$ are determined by requiring the fundamental TSh frequencies of an infinite plate calculated from the approximate plate equations and the exact three-dimensional equations to be equal. For a plate of monoclinic crystals, which includes rotated Y-cut quarts as a special case and is sufficient for purpose of this paper, the correction factors are [16]

$$
\kappa_{a}^{2}=\frac{\pi^{2}}{12} \frac{c_{a}\left\{1-C_{a} R_{\mathrm{S}}\left[1-C_{a} R_{\mathrm{S}}+\frac{1}{4} \pi^{2} C_{a}^{2}\left(R_{\mathrm{S}}^{2}-R_{\mathrm{D}}^{2}\right)\right]\right\}^{2}}{g_{2 a 2 a}\left[1+3 R_{\mathrm{S}}+3 R_{\mathrm{D}}^{2} /\left(1+R_{\mathrm{S}}\right)\right]}
$$

where

$$
\begin{aligned}
c_{1} & =c_{2121}, \\
c_{3} & =\frac{1}{2}\left\{c_{2222}+c_{2323}-\left[\left(c_{2222}-c_{2323}\right)^{2}+4 c_{2223}^{2}\right]^{1 / 2}\right\}, \\
C_{j} & =c_{j} / c_{2 j 2 j} .
\end{aligned}
$$

\section{Reduction to Extension And Elementary FlEXURE}

Eqs. (33) and (34) or (42) and (43) are coupled equations for the extension $u_{a}^{(0)}$, flexure $u_{2}^{(0)}$, and TSh $u_{a}^{(1)}$. For the applications we are interested in, $u_{a}^{(0)}, u_{2}^{(0)}$, and $u_{a}^{(1)}$ are biasing deformations resulting from $\tau_{a b}^{\prime}, E_{k}^{\prime}, H_{k}^{\prime}$ and similar fields from the lower film. For thin plates, $u_{a}^{(1)}$ is usually small and can be eliminated, resulting in a simpler theory for coupled extension and elementary flexure without shear. Such a theory will be sufficient for our needs and many other applications. It can be reduced from (33) and (34) as follows. First, we rewrite (33) and (34) as separate equations for $u_{a}^{(0)}, u_{2}^{(0)}$, and $u_{a}^{(1)}$ :

$$
\begin{gathered}
\mathcal{T}_{a b, a}^{(0)}+F_{b}^{(0)}=2 h \rho\left(1+R_{\mathrm{S}}\right) \ddot{u}_{b}^{(0)}+2 h^{2} \rho R_{\mathrm{D}} \ddot{u}_{b}^{(1)}, \\
\mathcal{T}_{a 2, a}^{(0)}+F_{2}^{(0)}=2 h \rho\left(1+R_{\mathrm{S}}\right) \ddot{u}_{2}^{(0)}, \\
\mathcal{T}_{a b, a}^{(1)}-\tau_{2 b}^{(0)}+F_{b}^{(1)}=0,
\end{gathered}
$$

where, as one of the two approximations needed for the reduction to elementary flexure, we have neglected the rotatory inertia terms on the right-hand side of (34). Eq. (48) now provides the usual shear force-bending moment relation in the elementary theory for flexure. Solving (48) for $\mathcal{T}_{2 b}^{(0)}$ and substituting the resulting expression into (47) gives the following equation for elementary flexure:

$$
\mathcal{T}_{a b, a b}^{(1)}+F_{b, b}^{(1)}+F_{2}^{(0)}=2 h \rho\left(1+R_{\mathrm{S}}\right) \ddot{u}_{2}^{(0)} .
$$

Another approximation needed for the reduction to elementary flexure is that the plate shear strains $S_{a 2}^{(0)}$ vanish, namely,

$$
S_{a 2}^{(0)}=\frac{1}{2}\left(u_{2, a}^{(0)}+u_{a}^{(1)}\right)=0 .
$$

This implies, through (9), that

$$
\begin{gathered}
u_{a}^{(1)}=-u_{2, a}^{(0)}, \\
S_{a b}^{(1)}=-u_{2, a b}^{(0)} .
\end{gathered}
$$

With (51) we can write the extensional equation (46) as

$$
\tau_{a b, a}^{(0)}+F_{b}^{(0)}=2 h \rho\left(1+R_{\mathrm{S}}\right) \ddot{u}_{b}^{(0)}-2 h^{2} \rho R_{\mathrm{D}} \ddot{u}_{2, b}^{(0)} .
$$

Eq. (53) and (49) are three equations for $u_{a}^{(0)}$ and $u_{2}^{(0)}$. The extension $u_{a}^{(0)}$ and the flexure $u_{2}^{(0)}$ are coupled on the righthand side of (53) when the two films are different. Because $S_{a 2}^{(0)}=0$ and $S_{22}^{(0)}$ is not present in (37), from (37) and (39) we have

$$
\begin{aligned}
\mathcal{T}_{a b}^{(0)}=2 h g_{a b c d}^{*} S_{c d}^{(0)} & +2 h\left(\gamma_{a b c d}^{\mathrm{S}} S_{c d}^{(0)}+h \gamma_{a b c d}^{\mathrm{D}} S_{c d}^{(1)}\right)+2 h N_{a b} \\
N_{a b}= & \frac{h^{\prime}}{h}\left(\tau_{a b}^{\prime}-e_{k a b}^{\prime} E_{k}^{\prime}-h_{k a b}^{\prime} H_{k}^{\prime}\right) \\
& +\frac{h^{\prime \prime}}{h}\left(\tau_{a b}^{\prime \prime}-e_{k a b}^{\prime \prime} E_{k}^{\prime \prime}-h_{k a b}^{\prime \prime} H_{k}^{\prime \prime}\right)
\end{aligned}
$$


The equations of motion for $u_{a}^{(0)}$ and $u_{2}^{(0)}$ are obtained by substituting (54) and (38) into (53) and (49). $S_{a b}^{(0)}$ and $S_{a b}^{(1)}$ are now given by (9) and (52), respectively, in terms of $u_{a}^{(0)}$ and $u_{2}^{(0)}$.

\section{Deformation of a Free Plate}

Consider the static deformation of a finite plate with traction-free surfaces all around. The top and bottom surface loads $F_{j}^{(0)}=0$ and $F_{b}^{(1)}=0$. The edge of the plate is geometrically smooth (without corners) and is free from any mechanical resultants. In this case, $\mathcal{T}_{a b}^{(0)} \equiv 0$ and $\mathcal{T}_{a b}^{(1)}$ $\equiv 0$ satisfy the governing equations in (53) and (49), as well as all boundary conditions. We consider the case of uniform intrinsic stresses and electric/magnetic fields in the films. In this case, $N_{a b}$ and $M_{a b}$ are constants. The corresponding constant plate extensional strains $S_{a b}^{(0)}$ and flexural strains $S_{a b}^{(1)}$ are determined by setting (54) and (38) to zero

$$
\begin{aligned}
\mathcal{T}_{a b}^{(0)} & =2 h g_{a b c d}^{*} S_{c d}^{(0)}+2 h\left(\gamma_{a b c d}^{\mathrm{S}} S_{c d}^{(0)}+h \gamma_{a b c d}^{\mathrm{D}} S_{c d}^{(1)}\right)+2 h N_{a b} \\
& =0 \\
\mathcal{T}_{a b}^{(1)}= & \frac{2}{3} h^{3} \gamma_{a b c d} S_{c d}^{(1)}+2 h^{3} \gamma_{a b c d}^{\mathrm{S}} S_{c d}^{(1)}+2 h^{2} \gamma_{a b c d}^{\mathrm{D}} S_{c d}^{(0)}+2 h M_{a b} \\
= & 0 .
\end{aligned}
$$

For elementary flexure $S_{2 a}^{(0)}=0 . S_{22}^{(0)}$ is determined from the stress relaxation condition $T_{22}^{(0)}=0 . S_{2 j}^{(1)}$ is determined from the stress relaxation conditions $T_{2 j}^{(1)}=0$. Then $S_{i j}^{(0)}$ and $S_{i j}^{(1)}$ are completely known. In the relatively simple case of cylindrical deformations of plates with $u_{3}=0$ and $\mathrm{\partial} / \mathrm{\partial} x_{3}=0$, from (56) and (57) we obtain

$$
\begin{aligned}
S_{1}^{(0)} & =\frac{-\gamma_{11}^{\mathrm{D}} M_{1}+h\left(\gamma_{11} / 3+\gamma_{11}^{\mathrm{S}}\right) N_{1}}{h\left(\gamma_{11}^{\mathrm{D}}\right)^{2}-h\left(\gamma_{11} / 3+\gamma_{11}^{\mathrm{S}}\right)\left(g_{11}^{*}+\gamma_{11}^{\mathrm{S}}\right)}, \\
S_{1}^{(1)} & =\frac{\left(g_{11}^{*}+\gamma_{11}^{\mathrm{S}}\right) M_{1}-h \gamma_{11}^{\mathrm{D}} N_{1}}{h^{2}\left(\gamma_{11}^{\mathrm{D}}\right)^{2}-h^{2}\left(\gamma_{11} / 3+\gamma_{11}^{\mathrm{S}}\right)\left(g_{11}^{*}+\gamma_{11}^{\mathrm{S}}\right)} .
\end{aligned}
$$

Eqs. (58) and (59) show that $\gamma_{11}^{D}$ causes coupling between extension and bending. For symmetric films, it vanishes.

\section{Frequency Shifts of the Fundamental Thickness-Shear Mode}

In this section, we consider TSh vibrations of a rotated Y-cut quartz plate with the presence of biasing fields, $S_{i j}^{(0)}$ and $S_{i j}^{(1)}$, caused by the intrinsic stresses and electric/magnetic fields in the surface films. The TSh vibration is an incremental motion superposed on these biasing fields. For the most widely used fundamental TSh mode, the frequency shift $\Delta \omega$ caused by the biasing fields is given by [18], [19]

$$
\begin{aligned}
\frac{\Delta \omega}{\omega_{0}}= & S_{1}^{(0)}+\frac{1}{2 c_{66}}\left(c_{166} S_{1}^{(0)}+c_{266} S_{2}^{(0)}+c_{366} S_{3}^{(0)}+c_{466} S_{4}^{(0)}\right) \\
& -\frac{h^{2}}{\sqrt{3} \pi c_{66}}\left(c_{165} S_{5,1}^{(1)}+c_{561} S_{1,3}^{(1)}+c_{563} S_{3,3}^{(1)}\right)
\end{aligned}
$$

where $\Delta \omega$ is normalized by the fundamental TSh frequency, $\omega_{0}$, of the crystal plate alone when the films are not present:

$$
\omega_{0}=\frac{\pi}{2 h} \sqrt{\frac{c_{66}}{\rho}} .
$$

$c_{661}$ and $c_{662}$ are the third-order elastic constants for nonlinear material behavior. They have six indices in the tensor notation and three indices under the compact matrix notation [20] used in (58). The first-order plate strains $S_{i j}^{(1)}$ have contributions only when they are inhomogeneous. In the special case of cylindrical deformations, (60) reduces to

$$
\frac{\Delta \omega}{\omega_{0}}=\left(1+\frac{c_{166}}{2 c_{66}}-\frac{c_{266} c_{21}}{2 c_{66} c_{22}}\right) S_{1}^{(0)} .
$$

\section{Examples of Application}

In this section, we use the equations derived in the preceding sections to study frequency shifts in a resonator caused by electric/magnetic fields and electrode stresses. Consider a crystal plate of Y-cut quartz, which is a special case of rotated Y-cuts in which the angle of rotation is zero. The linear material constants can be found in [20]. The third-order elastic constants are from [21]. The following relations among the third-order elastic constants exist and are needed [22]:

$$
\begin{aligned}
& c_{661}=\left(-2 c_{111}-c_{112}+3 c_{222}\right) / 4, \\
& c_{662}=\left(2 c_{111}-c_{112}-c_{222}\right) / 4 .
\end{aligned}
$$

For the plate thickness, we choose $2 h=1 \mathrm{~mm}$. The fundamental TSh mode of the plate alone without the surface films is $\omega_{0} / 2 \pi=1.940022 \times 10^{6} \mathrm{~Hz}$.

\section{A. Electric Field Sensing}

Consider the previously described quartz plate with only one surface film of PZT-5H (Sinocera Inc., Shanghai, China) poled in the thickness direction $\left(h^{\prime \prime}=0\right)$. Polarized ceramics have much stronger piezoelectric coupling than quartz. When the plate is placed under an external electric field in the thickness direction which we want to measure, the ceramic film tends to expand or contract but the quartz plate does not do so through piezoelectric coupling because its $e_{21}=e_{22}=e_{23}=0$. Instead, the quartz plate extends and bends under the action of the ceramic film. We solve (56) and (57) on a computer for $S_{a b}^{(0)}$ and $S_{a b}^{(1)}$, and 


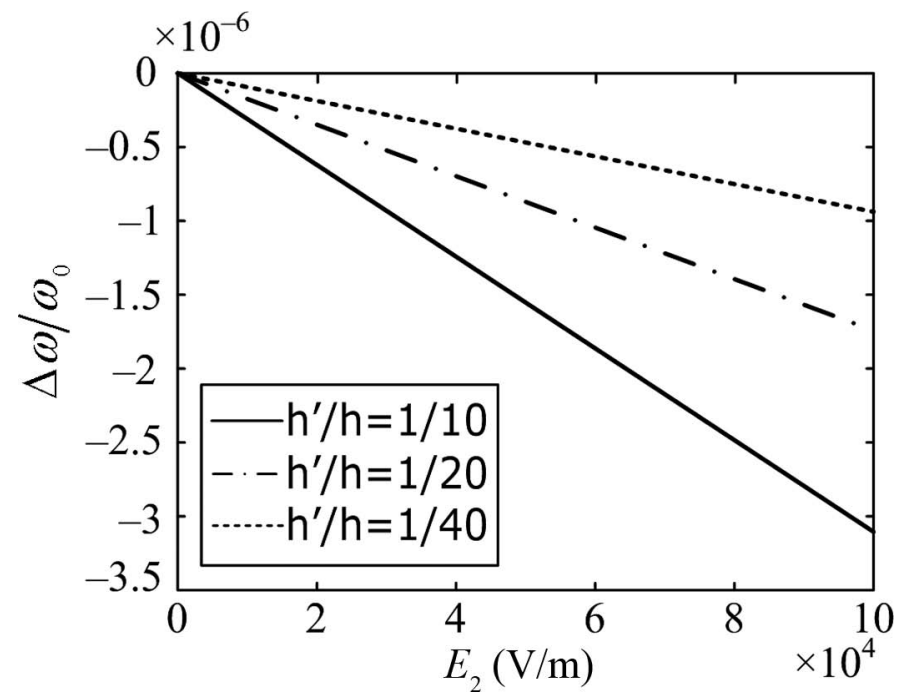

Fig. 2. Frequency shift resulting from electric field.

use the stress relaxation conditions to find the other plate strains. Then we calculate the frequency shift using (60) and plot the results in Fig. 2. A linear relationship between the frequency shift and the electric field is predicted, which is ideal for electric field sensing. This linearity is also a consequence of the theory employed, i.e., the biasing fields are assumed to be small and are obtained by the linear theory of elasticity, and only the first-order effect of the biasing fields on the incremental TSh vibration is considered. For large biasing fields, the nonlinear theory of elasticity is needed to determine the biasing fields, and the second- and higher-order effects of the biasing fields must be considered [23]. In that case, a nonlinear relationship between the frequency shift and the biasing fields would be predicted. Such a calculation requires the knowledge of the fourth-order material constants, which at present are not available; therefore, the range of the linear output cannot be determined from the present analysis. For a moderate electric field of $10^{5} \mathrm{~V} / \mathrm{m}$, or $100 \mathrm{~V} / \mathrm{mm}$, the relative frequency shift is of the order of $10^{-6}$, which is measurable in crystal resonators whose frequency shifts are typically described in parts per million. Fig. 2 shows that thicker piezoelectric films imply higher sensitivity, as expected. These results agree with reference [13] for cylindrical motions. Because the ceramic film is on one side of the crystal plate only, the electric field also causes bending of the crystal plate. The curvatures of the middle plane of the crystal plate are shown in Fig. 3 for $h^{\prime} / h=0.1$. The curvature in the $x_{3}$ direction is smaller because $c_{33}$ is larger than $c_{11}$ for Y-cut quartz plates.

\section{B. Magnetic Field Sensing}

When a Y-cut quartz plate carries only one piezomagnetic film $\left(h^{\prime \prime}=0\right)$ of $\mathrm{CoFe}_{2} \mathrm{O}_{4}$ (the material constants can be found in [24]), the analysis is similar. Quartz does not respond to magnetic fields directly. Through the extension or contraction of the piezomagnetic film, the quartz

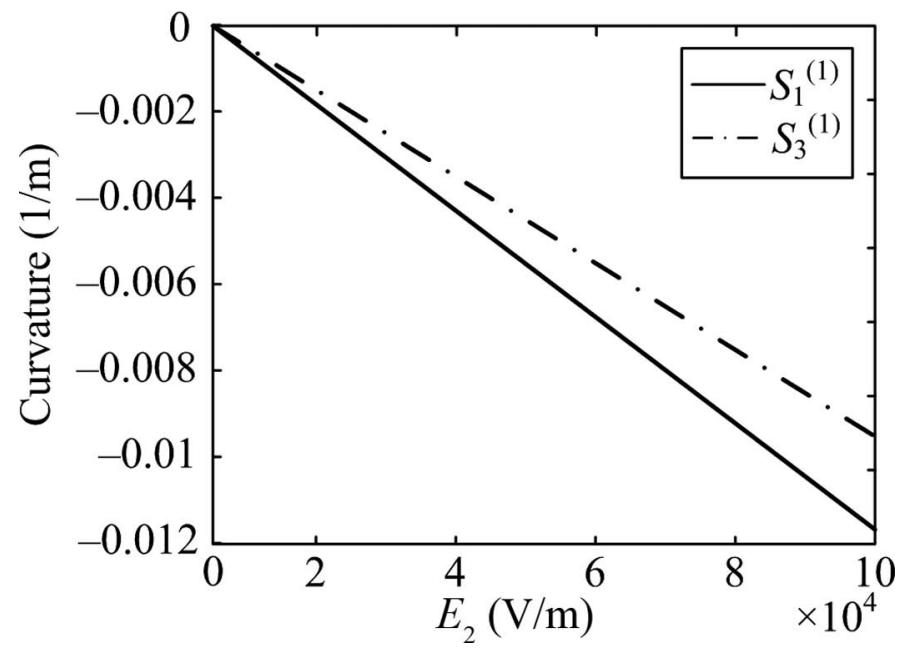

Fig. 3. Curvature resulting from electric field.

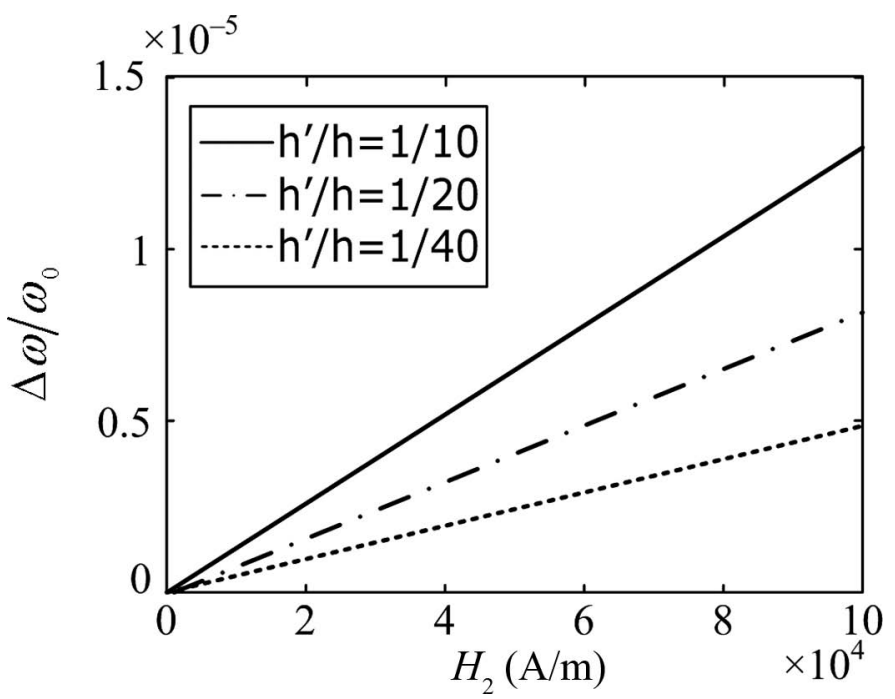

Fig. 4. Frequency shift resulting from magnetic field.

plate extends and bends, resulting in frequency shifts and curvatures. The results are shown in Figs. 4 and 5, respectively, showing that the structure can possibly function as a magnetic field sensor. These results also agree with the results of the cylindrical motions considered in [13].

\section{Electrode Stress}

Plate quartz resonators are usually exited by electrodes on the top and bottom of the plates. The electrodes often carry intrinsic stresses caused by their manufacturing processes. These electrode stresses are of the order of $200 \mathrm{MPa}$ [25]. Their effects on resonator frequency stability are an important issue in resonator design. As an example, consider a Y-cut quartz plate with identical electrodes of gold with intrinsic stresses of $200 \mathrm{MPa}$ in both the $x_{1}$ and $x_{3}$ directions. The frequency shift calculated from (60) is shown in Fig. 6. It is of the order of $10^{-5}$, quite significant.

Another important aspect of the electrodes is their thickness. In resonator manufacturing, one electrode is 


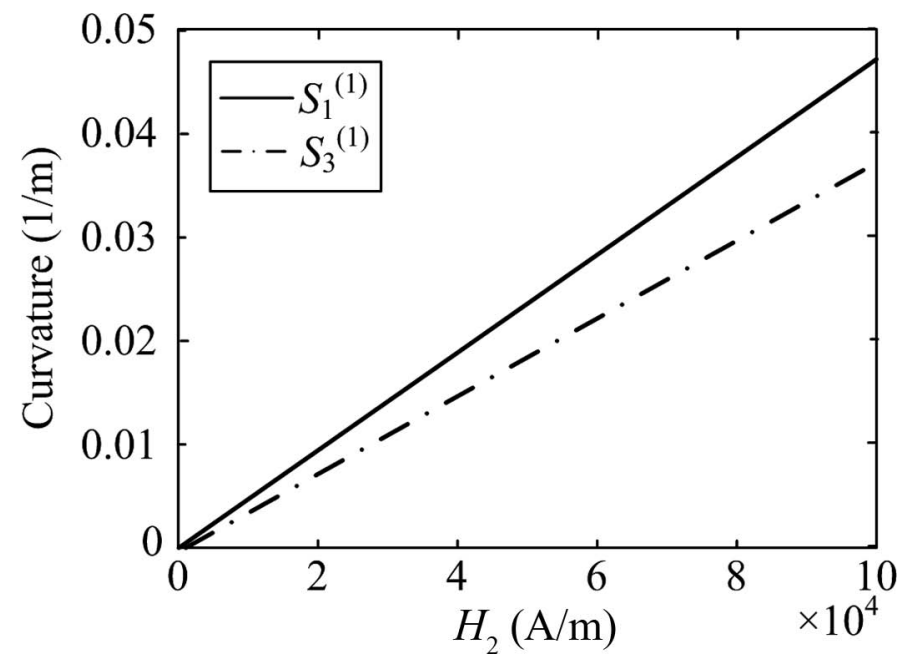

Fig. 5. Curvature resulting from magnetic field.

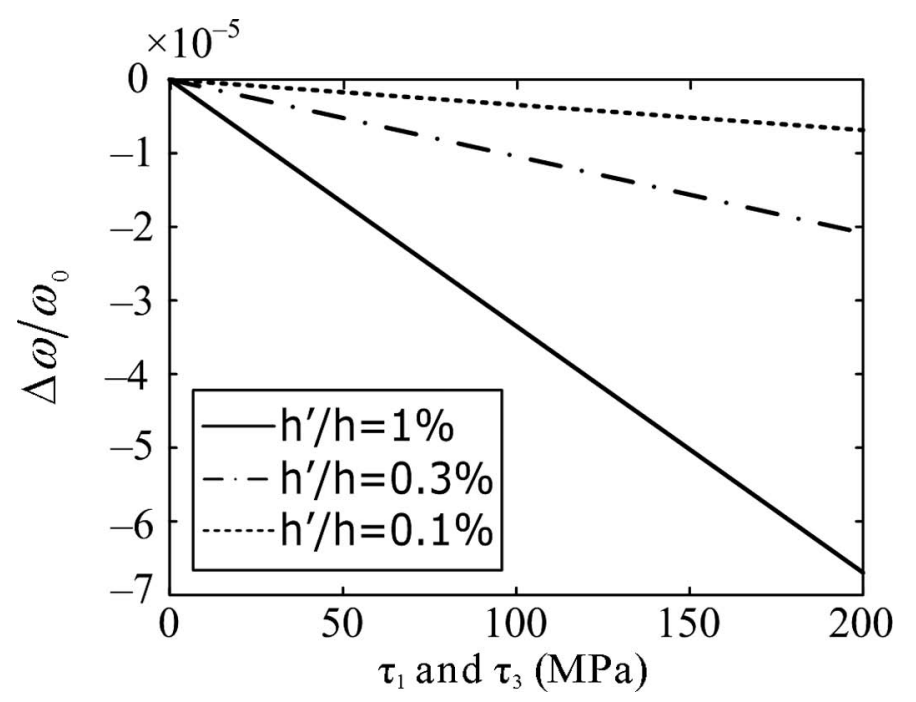

Fig. 6. Frequency shift resulting from identical electrode stress.

pre-deposited with a pre-determined thickness. The electrode on the other side of the plate has a thickness controlled by the desired frequency of the electroded plate. This normally results in a crystal plate with electrodes of unequal thickness. It is known that electrodes with different thickness cause undesirable mode couplings and affect resonator performance [16], [26]. As a numerical example, consider a Y-cut quartz plate with electrodes of different thickness. $h^{\prime} / h=0.003$ and $h^{\prime \prime} / h=0.001$. The corresponding curvatures are calculated from (56) and (57) and are shown in Fig. 7. If sufficient information about the plate strains and curvatures is measured experimentally, the electrode intrinsic stresses and/or their thicknesses can be calculated from measured data using (56) and (57).

\section{Conclusion}

Two-dimensional equations for coupled extension, flexure, and TSh motions of anisotropic crystal plates with

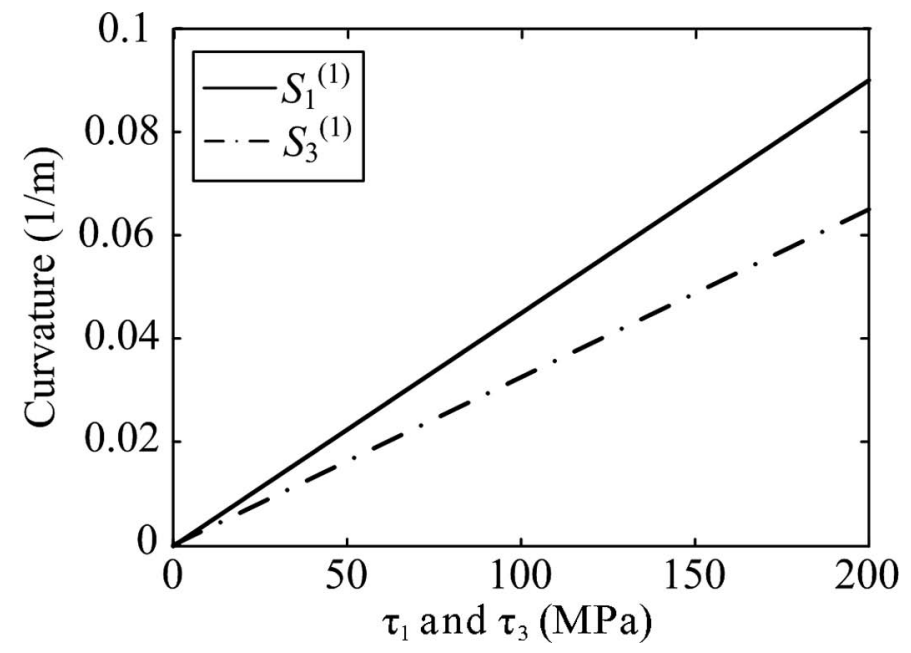

Fig. 7. Curvature resulting from electrode stress of unequal thickness.

multiphysical surface films are derived, reduced to couple extension and elementary flexure without shear, and used in the analysis of frequency shifts in crystal resonators. It is shown that a crystal plate with a piezoelectric/piezomagnetic film may be used as a sensor for electric/ magnetic fields. If the film/plate thickness ratio is $1 / 10$ and the electric field is of the order of $100 \mathrm{~V} / \mathrm{mm}$, the relative frequency shift is of the order of a few parts per million. If the magnetic field is of the order of $100 \mathrm{~A} / \mathrm{mm}$, the relative frequency shift is of the order of $10^{-5}$. These agree with some previous analyses in simpler situations, and are detectable frequency shifts in crystal resonators. The equations derived are also useful in the analysis of the effects of electrode stress in crystal resonators and other frequency analysis of plate crystal resonators and sensors in general.

\section{REFERENCES}

[1] H. F. Tiersten, "On the accurate description of piezoelectric resonators subject to biasing deformations," Int. J. Eng. Sci., vol. 33, no. 15, pp. 2239-2259, 1995.

[2] E. P. EerNisse, R. W. Ward, and R. B. Wiggins, "Survey of quartz bulk resonator sensor technologies," IEEE Trans. Ultrason. Ferroelectr. Freq. Control, vol. 35, no. 3, pp. 323-330, 1988.

[3] E. P. EerNisse, "Quartz resonators vs. their environment: Time base or sensor?" Jpn. J. Appl. Phys., vol. 40, no. 5B, pp. 3479-3483, 2001.

[4] E. Benes, "Improved quartz crystal microbalance technique," $J$. Appl. Phys., vol. 56, no. 3, pp. 608-626, 1984.

[5] E. Benes, M. Gröschl, W. Burger, and M. Schmid, "Sensors based on piezoelectric resonators," Sens. Actuators A Phys., vol. 48, no. 1, pp. 1-21, 1995.

[6] E. P. EerNisse, "Simultaneous thin-film stress and mass-change measurement using quartz resonators," J. Appl. Phys., vol. 43, no. 4, pp. 1330-1337, 1972.

[7] H. F. Tiersten, B. K. Sinha, and T. R. Meeker, "Intrinsic stress in thin films deposited on anisotropic substrates and its influence on the natural frequencies of piezoelectric resonators," J. Appl. Phys., vol. 52, no. 9, pp. 5614-5624, 1981.

[8] C. L. Zhang, W. Q. Chen, J. Y. Li, and J. S. Yang, "Two-dimensional analysis of magnetoelectric effects in multiferroic laminated plates," IEEE Trans. Ultrason. Ferroelectr. Freq. Control, vol. 56, no. 5, pp. 1046-1053, 2009.

[9] J. Willis and V. W. Lym, "Variable frequency quartz crystal resonators," IEEE Trans. Sonics Ultrason., vol. 12, no. 2, pp. 59-64, 1965. 
[10] J. C. Baumhauer and H. F. Tiersten, "Nonlinear electroelastic equations for small fields superposed on a bias," J. Acoust. Soc. Am., vol. 54 , no. 4, pp. 1017-1034, 1973.

[11] H. F. Tiersten, "On the nonlinear equations of thermo-electroelasticity," Int. J. Eng. Sci., vol. 9, no. 7, pp. 587-604, 1971.

[12] H. F. Tiersten and B. K. Sinha, "Temperature dependence of the resonant frequency of electroded doubly-rotated quartz thicknessmode resonators," J. Appl. Phys., vol. 50, no. 12, pp. 8038-8051, 1979.

[13] Y. Y. Zhou, W. Q. Chen, J. S. Yang, and J. K. Du, "Thickness-shear vibration of a quartz plate connected to piezoelectric plates and electric field sensing," Ultrasonics, vol. 51, no. 2, pp. 131-135, 2011.

[14] X. Y. Shan, Y. T. Hu, and J. S. Yang, "Reduction of surface acoustic wave resonator normal acceleration sensitivity using piezoelectric actuators," Appl. Phys. Lett., vol. 95, no. 12, art. no. 1235062009.

[15] H. Xue, Y. T. Hu, Q. M. Wang, and J. S. Yang, "Analysis of temperature compensation in a plate thickness mode bulk acoustic wave resonator," IEEE Trans. Ultrason. Ferroelectr. Freq. Control, vol. 54, no. 9, pp. 1826-1833, 2007.

[16] R. D. Mindlin, "High frequency vibrations of plated, crystal plates," in Progress in Applied Mechanics (the Prager Anniversary Volume). New York, NY: Macmillan, 1963, pp. 73-84.

[17] R. D. Mindlin, "High frequency vibrations of crystal plates," $Q$ Appl. Math., vol. 19, no. 1, pp. 51-61, 1961.

[18] P. C. Y. Lee, Y. S. Wang, and X. Markenscoff, "High-frequency vibrations of crystal plates under initial stresses," J. Acoust. Soc. Am., vol. 57, no. 1, pp. 95-105, 1975.

[19] P. C. Y. Lee, Y. S. Wang, and X. Markenscoff, "Nonlinear effects of initial bending on the vibrations of crystal plates," J. Acoust. Soc. Am., vol. 59, no. 1, pp. 90-96, 1976.

[20] H. F. Tiersten, Linear Piezoelectric Plate Vibrations. New York, NY: Plenum, 1969.

[21] R. N. Thurston, H. J. McSkimin, and P. Andreatch Jr., "Thirdorder elastic constants of quartz," J. Appl. Phys., vol. 37, no. 1, pp. 267-275, 1966 .

22] D. F. Nelson, Electric, Optic and Acoustic Interactions in Crystals New York, NY: Wiley, 1979, pp. 481-513.

[23] J. S. Yang and J. A. Kosinski, "Perturbation analysis of frequency shifts in an electroelastic body under biasing fields," IEEE Trans. Ultrason. Ferroelectr. Freq. Control, vol. 53, no. 12, pp. 2442-2449, 2006.

[24] F. Ramirez, P. R. Heyliger, and E. N. Pan, "Free vibration response of two-dimensional magneto-electro-elastic laminated plates," $J$ Sound Vibrat., vol. 292, no. 3-5, pp. 626-644, 2006.

25] E. P. EerNisse "Quartz resonator frequency shifts arising from electrode stress," in Proc. 29th Frequency Control Symp., 1975, pp. 1-4.

[26] J. N. Wang, Y. T. Hu, and J. S. Yang, "Frequency spectra of AT-cut quartz plates with electrodes of unequal thickness," IEEE Trans. Ultrason. Ferroelectr. Freq. Control, vol. 57, no. 5, pp. 1146-1151, 2010.
Nan Liu's photograph and biography were not available at time of publication.

Jiashi Yang's photograph and biography were not available at time of publication.

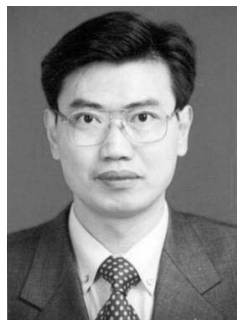

Yuantai $\mathbf{H u}$ received his B.S. degree in mining engineering in 1986 from Wuhan University of Technology, Wuhan, China, his M.S. degree in mechanics from Harbin University of Technology, Harbin, China, in 1988, and his Ph.D. degree in mechanics from the Shanghai Institute of Applied Mathematics and Mechanics, Shanghai University, Shanghai, China, in 1992. His research interests are piezoelectric structures and devices, ultrasonics, and acoustics.

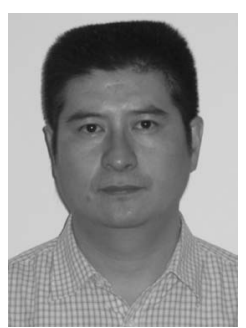

Xuedong Chen received his B.S. and M.S. degrees in mechanical engineering from Wuhan University of Technology, Wuhan, China, in 1984 and 1989, respectively, and his Ph.D. degree from Saga University, Saga, Japan, in 2001. His research interests include robotics, mechanical dynamics, vibration and control, etc.

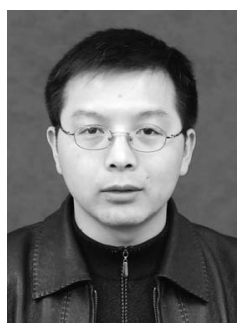

Wei Jiang received his B.S. and Ph.D. degrees in mechanical engineering from Huazhong University of Science and Technology, Wuhan, China, in 2002 and 2008. His research interests include mechanical dynamics, vibration and control, etc. 